\title{
Analysis of real-time three dimensional transesophageal echocardiography in the assessment of left atrial appendage function in patients with atrial fibrillation
}

\author{
LIN GAN, LAN YU, MANYING XIE, WEI FENG and JIABAO YIN \\ Department of Ultrasound Imaging, Xiangyang Hospital Affiliated to Hubei University of Medicine, \\ Xiangyang, Hubei 441000, P.R. China
}

Received March 17, 2016; Accepted September 20, 2016

DOI: $10.3892 / \mathrm{etm} .2016 .3745$

\begin{abstract}
The aim of the study was to examine changes in left atrial appendage volume (LAA-V) in patients with non-valvular atrial fibrillation (AF) using real-time threedimensional transesophageal echocardiography (RT3D-TEE) and evaluate the prediction value on the high risk of thrombosis of LAA. Using RT3D-TEE we measured: i) LAA peak empty velocity (LAA-PEV), ii) LAA-V including LAA end-diastolic volume (LAA-EDV) and end-systolic volume (LAA-ESV). We also calculated LAA ejection fraction (LAA-EF). RT3D-TEE was applied in 20 control cases and 74 patients with non-valvular AF. According to the presence of thrombosis, 74 patients were divided into the no thrombosis group (NTH group, $\mathrm{n}=52$ ) and thrombosis group (TH group, $n=22$ ). Our results showed that there were significant differences in LAA-V and LAA-EF values in different groups $(\mathrm{P}<0.05)$. LAA-EDV moderately correlated with LAA-PEV $(\mathrm{r}=-0.531, \mathrm{P}<0.001)$ while LAA-ESV demonstrated a strong correlation with LAA-PEV ( $r=-0.741$, $\mathrm{P}<0.001)$. LAA-EF also showed a strong correlation with LAA-PEV ( $\mathrm{r}=0.693, \mathrm{P}<0.001)$. Through receiver operating characteristic (ROC) curves, the cut-off values of LAA-EDV and LAA-ESV in thrombosis of LAA were 18.45 and $9.69 \mathrm{ml}$, respectively. RT3D-TEE effectively evaluated the LAA-V, LAA-PEV and LAA-EF parameters, and proved to be valuable in the process of evaluation of thrombosis of LAA.
\end{abstract}

\section{Introduction}

Atrial fibrillation (AF) is a condition involving an irregular heart rhythm, known as arrhythmia. It is the most common type

Correspondence to: Dr Jiabao Yin, Department of Ultrasound Imaging, Xiangyang Hospital Affiliated to Hubei University of Medicine, 15 Jiefang Road, Xiangyang, Hubei 441000, P.R. China E-mail: yin_jiabao1@163.com

Key words: real-dimension echocardiography, echocardiography, transesophagy, atrial fibrillation of arrhythmia (1). The most common conditions leading to AF are: i) rheumatic valvular disease, ii) coronary heart disease, and iii) hypertensive heart disease. AF is the main risk factor inducing cardiogenic strokes (2). Increased pressure and blood stagnation occurred in AF, due to the particular anatomical structure of the left atrial appendage, which is the narrowest part in the left atrium with the roughest endocardial surface. Therefore thrombus easily occurs in the left atrial appendage, and in previous studies, length, width, emptying rate and other indicators were employed to evaluate its function (3).

In the present study, we measured the left atrial appendage volume (LAA-V) through real-time three-dimensional transesophageal echocardiography (RT3D-TEE) to calculate LAA ejection fraction (LAA-EF). Subsequently, the association between the changes in LAA-V, LAA-EF and thrombosis when LAA occurred was analyzed. These analyses provided valuable information pertaining to patients with a high risk of thrombosis and guided our clinical treatment and reduced the occurrence of LAA and cardiogenic stroke.

\section{Materials and methods}

Research object. From September, 2014 to December, 2014, 74 patients diagnosed with AF at the Xiangyang Hospital Affiliated to Hubei University of Medicine (Hubei, China), were selected. Patients with valvular heart diseases were excluded. There were 32 men and 42 women, with an age range of 34-76 years (average, 58.86 \pm 9.85 years). Of the 74 cases, 26 suffered from persistent AF while 48 had paroxysmal AF. According to the results obtained through ultrasonic cardiogram and clinical examinations all 74 cases were diagnosed with AF. There were 3 groups: i) the LAA thrombosis group (TH group) with 22 cases, ii) the non-LAA thrombosis group (NTH group) with 52 cases, and iii) the control group with 20 randomly chosen patients from our hospital. Patients in the control group comprised 10 men and 10 women (average age, $44.21 \pm 12.29$ years), who did not have AF.

Apparatus and method. Philips iE33 color Doppler ultrasonic diagnostic apparatus was used (Philips, Eindhoven, The Netherlands). Patients were detected using TTE, connected to the electrocardiogram, to have the information related to 


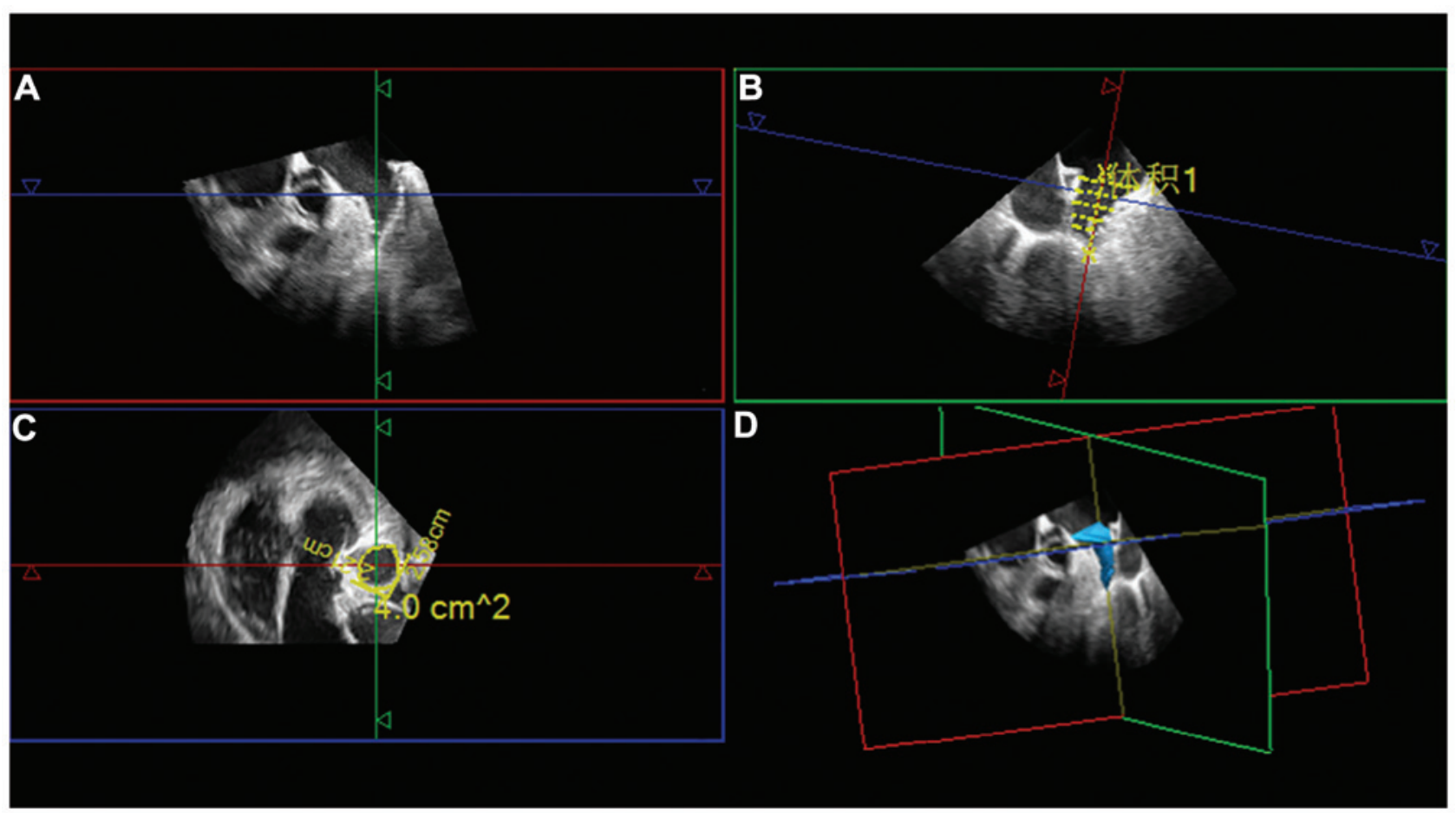

Figure 1. Measurement of LAA-V by GI3DQ. (A-C) Three orthogonal planes of LAA-V, (A and B) the long axis cross section of LAA-V, (C) the cross section of LAA-V, and (D) three-dimensional image of LAA-V. LAA-V, left atrial appendage volume.

their heart size, morphology, structure and function. Informed consent was obtained from the patients. Approval of the study was obtained by the ethics committee of the Xiangyang Hospital Affliated to Hubei University of Medicine. TEE (X7-2t probe, 2-7 MHz) was used. After TEE, patients fasted for $>8 \mathrm{~h}$ with no water for $>4 \mathrm{~h}$. In cases with dentures they were removed at the same time. On the examination of TEE, the patients with local anesthesia of lidocaine hydrochloride were examined in the left lateral decubitus position, biting mouthparts were placed in their oral cavity, and probe $X 7-2 t$ was sent into the esophagus to scan each section of left atrial appendage in the middle of the esophagus, to observe thrombosis and measure LAA peak empty velocity (LAA-PEV). Then full-volume was initiated, five cardiac cycles were stored, and QLAB 9.0 software (Philips Medical System, Andover, MA, USA) was applied in the analysis.

\section{Measurement index}

$L A A-P E V$. In the middle of the TEE esophagus, approximately $45^{\circ}$, a long axis of the left atrial appendage was displayed, a pulse-waved (PW) Doppler was applied in placing the sample volume at the central position with $<1 \mathrm{~cm}$ the distance from the left atrial appendage entrance to obtain a spectrogram, and forward wave in the beginning of $\mathrm{P}$-wave in patients with sinus rhythm, the peak velocity of which was LAA-PEV. In the AF patients, the average value of maximum forward velocity in each cardiac cycle of five cardiac cycles was LAA-PEV.

$L A A$ end-diastolic volume (LAA-EDV), LAA end-systolic volume ( $L A A-E S V)$ and $L A A-E F$. The full-volume image was opened by GI3DQ plugin and the target area was adjusted, as there were three perpendicular sections of LAA, two long axis section and a transverse section (Fig. 1A-C). One of the long axis sections was selected, stacked contours button was clicked, and the software automatically measured the vertical distance from the entrance to the top of LAA. A long axis section was cut into several layers of equidistant cross sections (adjustable 3-16 layers, Fig. 1B), and the contour of each depicting cross section was shown. The instrument then automatically generated the 3D image of LAA and computed the LAA-V (Fig. 1D).

The measurement of LAA-EDV and LAA-ESV. Patients with sinus rhythm were detected, respectively, in the beginning of P-wave and in the end of QRS wave. The mean values of the maximum volume and the minimum volume in each cardiac cycle of 5 consecutive cardiac cycles were measured in patients with AF. Subsequently, LAA-EF was calculated using the following formula: (LAA-EDV-LAA-ESV)/(LAA-EDV).

Statistical analysis. SPSS 18.0 software package (Chicago, IL, USA) was used for data processing. Data were expressed as mean \pm standard deviation (SD). One-way analysis of variance (ANOVA) was employed in the comparison of multiple sets of measurement data. LSD methods were used to compare between two mean values of multiple sets of mean values. Pearson's method was applied to compare LAA-PEV, LAA-V and LAA-EF. Receiver operating characteristic (ROC) curve was used in the prediction of cut-off values of the left atrial appendage thrombus through LAA-EDV and LAA-ESV. $\mathrm{P}<0.05$ was considered statistically significant.

\section{Results}

Left atrial thrombosis. Of the 74 patients with AF, 22 cases had LAA thrombus by TEE, flat or papillary, 2.0x3.3 mm to 
Table I. Measurement value of the left atrial appendage in the three groups.

\begin{tabular}{lccccc}
\hline Groups & Cases & LAA-PEV $(\mathrm{cm} / \mathrm{s})$ & LAA-EDV $(\mathrm{ml})$ & LAA-ESV $(\mathrm{ml})$ & LAA-EF $(\%)$ \\
\hline Control group & 20 & $77.08 \pm 21.02$ & $9.18 \pm 2.54$ & $2.41 \pm 1.41$ & $74.50 \pm 12.03$ \\
NTH group & 52 & $70.28 \pm 32.58$ & $13.56 \pm 3.69^{\mathrm{a}}$ & $5.58 \pm 2.24^{\mathrm{a}}$ & $59.53 \pm 9.55^{\mathrm{a}}$ \\
TH group & 22 & $43.90 \pm 22.09^{\mathrm{a}, \mathrm{b}}$ & $20.61 \pm 5.74^{\mathrm{a}, \mathrm{b}}$ & $10.72 \pm 3.93^{\mathrm{a}, \mathrm{b}}$ & $47.82 \pm 15.44^{\mathrm{a}, \mathrm{b}}$ \\
F-value & & 5.933 & 20.467 & 26.069 & 12.712 \\
P-value & & 0.005 & $<0.001$ & $<0.001$ & $<0.001$ \\
\hline
\end{tabular}

Compared with the control group, ${ }^{\mathrm{a}} \mathrm{P}<0.05$; compared with the $\mathrm{NTH}$ group, ${ }^{\mathrm{b}} \mathrm{P}<0.05$. LAA-PEV, left atrial appendage peak empty velocity; LAA-EDV, left atrial appendage end-diastolic volume; LAA-ESV, left atrial appendage end-systolic volume; LAA-EF, left atrial appendage ejection fraction.

$8.9 \times 5.7 \mathrm{~mm}$. Of the 22 cases, 14 cases were fresh thrombus with weak and low echo, and 8 cases had organized thrombus with strong echo (Fig. 2), 10 cases were located in the pectinate muscles at the top of LAA, 7 cases were located on the lateral wall of LAA, and 5 cases were located on the medial wall of left atrial appendage. After thrombolytic therapy for 3-5 months, LAA thrombus of 14 patients disappeared through TEE review, and left atrial appendage thrombus of 8 patients was significantly shrunk.

Comparison of ultrasonic measurement values of LAA. The comparison of parameters in the three groups is shown in Table I. Differences between the LAA-PEV and LAA-EF, NTH group and TH group were statistically significant $(\mathrm{P}<0.05)$. Differences between LAA-EDV and LAA-ESV in all 3 groups were statistically significant $(\mathrm{P}<0.05)$.

Association between LAA-PEV and other measurement values. LAA-EDV and LAA-PEV showed a moderate negative correlation $(\mathrm{r}=-0.531, \mathrm{P}<0.001)$. LAA-PEV and LAA-ESV showed a strong negative correlation $(\mathrm{r}=-0.741, \mathrm{P}<0.001)$, while LAA-EF and LAA-PEV had a strong positive correlation $(\mathrm{r}=0.693, \mathrm{P}<0.001)$.

ROC curve analysis of $L A A-V$. The cut-off values for the forecast of thrombus by LAA-EDV and LAA-ESV were 18.45 and $9.69 \mathrm{ml}$, area under the curve was 0.896 and $0.807,95 \%$ confidence interval was $0.814-0.978$ and $0.693-0.920$, respectively $(\mathrm{P}<0.05)$ (Fig. 3).

\section{Discussion}

LAA is the remnants of primitive atrium in embryonic period, a narrow and curved pipe, the spire is a narrow blind side, and the body is rich in myocardial cells. These special anatomical features lead to thrombus formed in the left atrial appendage rather than in atrium sinistrum and other structures in left atrial blood flow deposition (4,5). The most common causes of inducing left atrial blood flow deposition are AF. In AF, the coordination between atrial and ventricular is broken. This is combined with the non-coordinated atrial contraction, which induces the atrial blood ejection deficiency and blood flow deposition, and eventually leads to higher atrial volume and pressure (6).

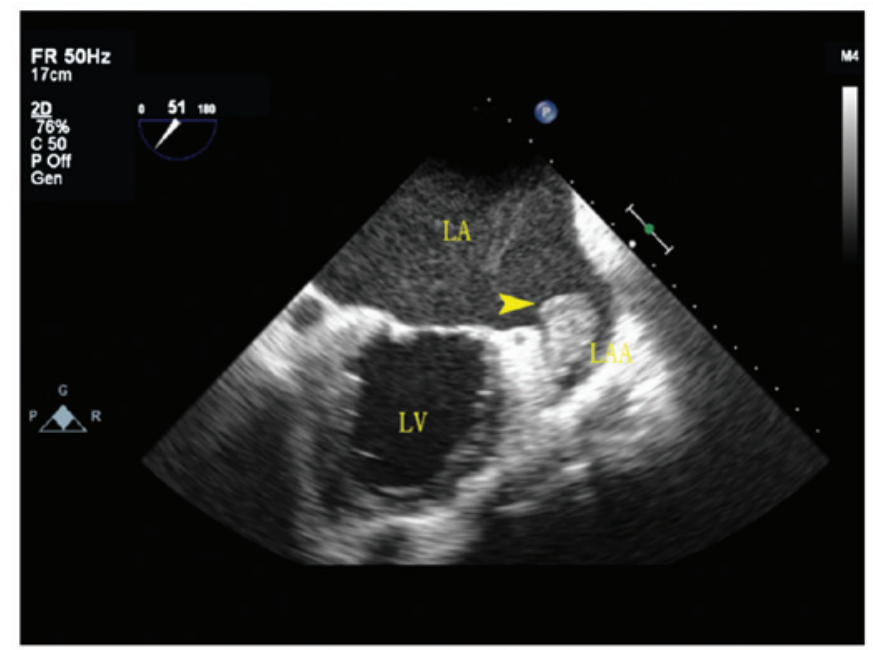

Figure 2. Left atrial thrombus. LA, atrium sinistrum; LV, ventriculus sinister; LAA, left atrial appendage; the yellow arrow, left atrial appendage thrombus.

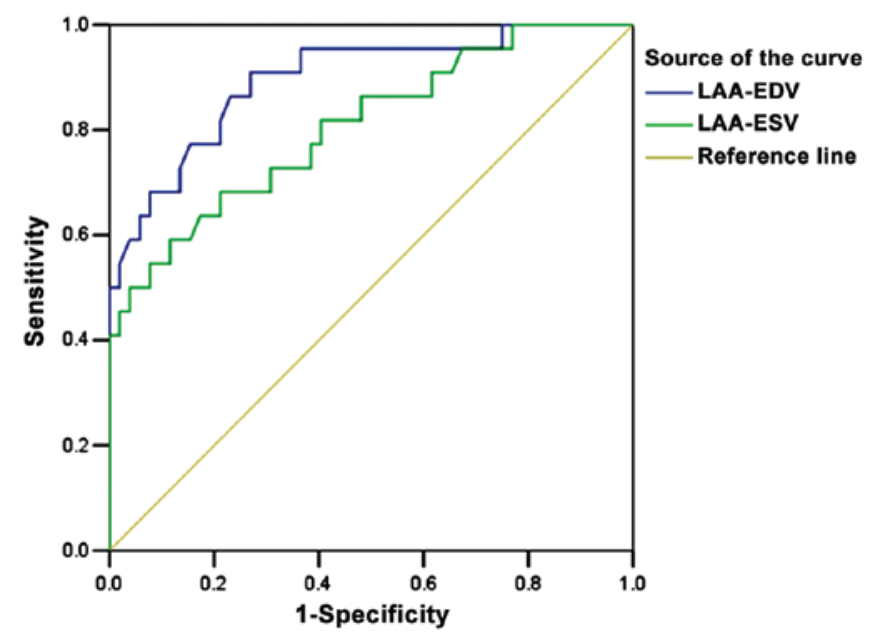

Figure 3. The prediction of ROC curve of LAA thrombosis by LAA-EDV and LAA-ESV. ROC, receiver operating characteristic; LAA, left atrial appendage; LAA-EDV, LAA end-diastolic volume; LAA-ESV, LAA end-systolic volume.

In the case of LAA-V, it is believed that ' $t$ ' can be used as a predictive value of thromboembolic stroke for patients 
with AF (7), and studies have found that increased LAA-V and decreased LAA-EF were significantly correlated with paroxysmal AF. The variability of LAA-V observed through the detection of R3D-TEE was small (8). The present study used GI3DQ of Q-lab software to respectively calculate LAA-EDV and LAA-ESV, then calculate LAA-EF. A comparison of LAA-V in all three groups, showed that the control group had the lowest LAA-V and the TH had the highest. Differences were statistically significant. These results indicated that $\mathrm{LAA}-\mathrm{V}$ increased in AF, and with the increased LAA-V, the risk of suffering from LAA thrombus was also increased. The increase of LAA-V in patients with AF may be due to an increase in left auricle cardiomyocytes in AF and loss of muscle fiber in the cells, which decreased left atrial systolic function, reduced LAA blood ejection (9) and shortened left atrial diastolic and systolic time. The two factors lead to blood stagnation of the left atrial appendage and increased LAA-V. LAA-V was usually only approximately $10 \%$ of the left atrium, but it played an important role in the left atrium function. Compared with the intrinsic atrial, the compliance of LAA was improved; thus, when the pressure and volume of the left atrial increased, LAA acted as a reservoir. This was the reason for the intrinsic atrial becoming only slightly enlarged in patients with AF, while LAA became significantly expanded (10). In the group of patients with AF combined with left atrial thrombus, conventional transthoracic echocardiography did not show the thrombus. Therefore, conventional TEE examinations in patients with AF were very important.

Previous findings confirmed that formation of LAA thrombus, significantly decreased LAA-PEV (11). A low LAA-PEV value has been proven to increase the risk of LAA thrombus (12). In clinical examinations, we often evaluated the function of the left atrial appendage in patients with AF by measuring LAA-PEV. It is believed that LAA-PEV are independent risk factors in the formation of LAA thrombus (13). The present study has shown that LAA-V and LAA-PEV were negatively correlated and changes in LAA function reflected by LAA-V and hemodynamics changes reflected by LAA-PEV were similar. Additionally, according to the ROC curve, and area under the curve (AUC), the cut-off values by LAA-ESV and LAA-EDV were 0.807 and 0.896 , respectively, which indicated that use of LAA-V in the assessment of risk of LAA thrombosis had a higher accuracy. Results obtained in the present study were similar with results reported by Tanaka et al on the relationship between LAA-VI and the occurrence of paroxysmal AF in patients with cerebral infarction (8). Tanaka et al evaluated the cut-off point of paroxysmal AF with LAA-VI and obtained higher AUC (8).

Previous findings showed that, compared with the control group, the LAA-EF of patients with non-valvular AF was significantly lower (14). In the present study, the comparison of LAA-EF in three groups through LAA-V showed that, the control group was the highest and the TH group was the lowest. This indicated that the left fibrillation systolic function of patients with fibrillation decreased, and with the reduced systolic function, the risk of thrombus increased. Left fibrillation systolic function was an important factor in affecting left atrial ejection (15); thus, the study on the relationship of LAA-EF and LAA-PEV was significant. This study found that LAA-EF had a strong positive correlation with LAA-PEV, which showed that the measurement of LAA-EF with RT3D-TEE in assessment of left fibrillation function was feasible. Through measurement of the left fibrillation function in patients in acute stage of cerebral infarction, it was found that LAA-EF and LAA-PEV in the paroxysmal AF groups were significantly lower than those of non-AF groups. Through the ROC curve, it was hypothesized that, LAA-EF $<49.1 \%$ was able to predict the occurrence of paroxysmal AF (16), which provided more information for clinical prognosis and active treatment.

In conclusion, application of RT3D-TEE identified the structure of LAA comprehensively in real-time and evaluated the function of LAA through the measurement of LAA-PEV, LAA-EDV, LAA-ESV and LAA-EF. This method can be used to evaluate the prognosis of patients with non-valvular $\mathrm{AF}$, and reduce the occurrence of cardiac stroke.

\section{References}

1. Nattel S, Li D and Yue L: Basic mechanisms of atrial fibrillation - very new insights into very old ideas. Annu Rev Physiol 62: 51-77, 2000.

2. Iwahana H, Ishikawa S, Ishikawa J, Kabutoya T, Kayaba K, Gotoh T and Kajii E: Atrial fibrillation is a major risk factor for stroke, especially in women: the Jichi Medical School cohort study. J Epidemiol 21: 95-101, 2011.

3. Wysokinski WE, Ammash N, Sobande F, Kalsi H, Hodge D and McBane RD: Predicting left atrial thrombi in atrial fibrillation. Am Heart J 159: 665-671, 2010.

4. Burrell LD, Horne BD, Anderson JL, Muhlestein JB and Whisenant BK: Usefulness of left atrial appendage volume as a predictor of embolic stroke in patients with atrial fibrillation. Am J Cardiol 112: 1148-1152, 2013.

5. Habara S, Dote K, Kato M, Sasaki S, Goto K, Takemoto H, Hasegawa D and Matsuda O: Prediction of left atrial appendage thrombi in non-valvular atrial fibrillation. Eur Heart J 28: 2217-2222, 2007.

6. Stoddard MF, Dawkins PR, Prince CR and Ammash NM: Left atrial appendage thrombus is not uncommon in patients with acute atrial fibrillation and a recent embolic event: A transesophageal echocardiographic study. J Am Coll Cardiol 25: 452-459, 1995

7. Santiago D, Warshofsky M, Li MG, Di Tullio M, Coromilas J, Reiffel $J$ and Homma S: Left atrial appendage function and thrombus formation in atrial fibrillation-flutter: A transesophageal echocardiographic study. J Am Coll Cardiol 24: 159-164, 1994

8. Tanaka K, Koga M, Sato K, Suzuki R, Minematsu K and Toyoda K: Three-dimensional analysis of the left atrial appendage for detecting paroxysmal atrial fibrillation in acute ischemic stroke. Int J Stroke 9: 1045-1051, 2014.

9. Li AL, Li ZA, Wang Y, Zeng YJ and Sun CL: Assessment of left atrial appendage function by real-time three-dimensional transesophageal echocardiography. Chinese J Ultrasonography 19: 737-740, 2010.

10. Nakajima H, Seo Y, Ishizu T, Yamamoto M, Machino T, Harimura Y, Kawamura R, Sekiguchi Y, Tada H and Aonuma K: Analysis of the left atrial appendage by three-dimensional transesophageal echocardiography. Am J Cardiol 106: 885-892, 2010.

11. Demirçelik MB, Çetin M, Çiçekcioğlu H, Uçar Ö and Duran M: Effect of left ventricular diastolic dysfunction on left atrial appendage function and thrombotic potential in nonvalvular atrial fibrillation. Anadolu Kardiyol Derg 14: 256-260, 2014

12. Zabalgoitia M, Halperin JL, Pearce LA, Blackshear JL, Asinger RW and Hart RG: Transesophageal echocardiographic correlates of clinical risk of thromboembolism in nonvalvular atrial fibrillation. Stroke prevention in atrial fibrillation III investigators. J Am Coll Cardiol 31: 1622-1626, 1998. 
13. Zateyshchikov DA, Brovkin AN, Chistiakov DA and Nosikov VV: Advanced age, low left atrial appendage velocity, and factor $\mathrm{V}$ promoter sequence variation as predictors of left atrial thrombosis in patients with nonvalvular atrial fibrillation. $\mathrm{J}$ Thromb Thrombolysis 30: 192-199, 2010.

14. Bi WJ, Sun FF, Ren WD, Pan FZ, Hu Q and Xu M: Real-time three-dimensional transesphageal echocardiography in evaluaying on morphology and mechanical function of left atrial appendage in patients with non-valvular atrial fibrillation. Chinese Journal of Medical Imaging Technology 10: 1616-1620, 2013.
15. Meng FX, Chen M, Sun JP and Dong Y: The assessment of left atrial appendage function in patients at high risk of thrombosis through transesophageal echocardiography. Chinese J Medical Imaging Technol 6: 473-479, 2014.

16. Shimizu T, Takada T, Shimode A, Fujita Y, Usuki N, Kato B, Takaishi S, Hirayama T, Hanzawa K and Hasegawa Y: Association between paroxysmal atrial fibrillation and the left atrial appendage ejection fraction during sinus rhythm in the acute stage of stroke: a transesophageal echocardiographic study. J Stroke Cerebrovasc Dis 22: 1370-1376, 2013. 